\title{
Bleomycin Induced Drug Allergy Mimicking Herpes Skin Infection: A Case Report
}

\author{
Zaw Thant Lwin, Zen Yong \\ Department of Acute Internal Medicine, Ninewells Hospital, Dundee, UK \\ Email: zaw.lwin@nhs.net, zen.yong@nhs.net
}

How to cite this paper: Lwin, Z.T. and Yong, Z. (2020) Bleomycin Induced Drug Allergy Mimicking Herpes Skin Infection: A Case Report. Open Journal of Blood Diseases, 10, 55-60.

https://doi.org/10.4236/ojbd.2020.102007

Received: May 10, 2020

Accepted: June 13, 2020

Published: June 16, 2020

Copyright (อ 2020 by author(s) and Scientific Research Publishing Inc. This work is licensed under the Creative Commons Attribution International License (CC BY 4.0).

http://creativecommons.org/licenses/by/4.0/

\begin{abstract}
This is a case report of bleomycin induced drug allergy in a 34-year-old gentleman. He developed generalized maculopapular rashes with some vesicles over the shoulders, abdomen, both upper limbs and right thigh on the second day after administration of bleomycin and that can be mistaken for herpes skin infections if we do not perform clinical examination thoroughly. In this case report, the importance of distinguishing between herpes virus skin infection and drug induced reaction is emphasized and the differences in management strategies are highlighted.
\end{abstract}

\section{Keywords}

Bleomycin, Maculopapular Rashes, Anaphylactic Reaction

\section{Introduction}

Bleomycin is an anti-cancer chemotherapy drug. It is also classified as an antitumor antibiotic. It is chiefly given as an infusion into the vein. Bleomycin containing chemotherapy regimen is highly employed and effective in treating Hodgkin lymphoma disease. One of the serious side effects but uncommon one is severe allergic reaction (anaphylaxis) that happens immediately or after several hours. A severe reaction such as low blood pressure, confusion, fever, chills, and wheezing may occur in few patients treating lymphoma. If this is to occur, it will generally occur after the first or second dose. In this case, the standard type of chemotherapy regimen should not be used any longer and it is necessary to consider another type of chemotherapy regimen and to avoid the use of bleomycin. In addition, clinicians should identify the type of drug reaction early and prompt action should be taken seriously. 


\section{Case Report}

A 34-year-old gentleman presented with maculopapular rashes over both shoulders, abdomen, both upper limbs [Figure 1] including palms [Figure 2] and right shin [Figure 3] two days after ABVD chemotherapy regimen for his type II A Hodgkin lymphoma. His main complaints were itchiness and small vesicles over the lesion but not pain. Initially, He was diagnosed as herpes skin infection and treated with IV acyclovir for 3 days. However, it did not show any sign of improvement. Hence, we decided to stop the antiviral treatment observing him clinically for a few days in the hospital and he was discharged following the clinical improvement.

One week later he was reviewed in hematology clinic for another cycle of the same chemotherapy regimen named 1 B ABVD chemo. A day later he was admitted to hospital again for similar complaints but worse than before: generalized skin rashes with swelling of both eyes accompanied by intense itchiness. However, there was no mucosal involvement in the oral cavity. At that point, our impression was bleomycin induced drug reaction rather than herpes skin infection. High dose steroids and antihistamine treatment were provided to the patient and there was a significant response to the treatment. Decision thereafter was not to employ bleomycin any longer as part of the chemotherapy regime.

On subsequent visits, his skin rashes improved significantly and only brown Colored skin was left. Since he received another type of chemotherapy regimen to avoid bleomycin, no similar problems occurred to him and he went through well with the new chemotherapy regimen.

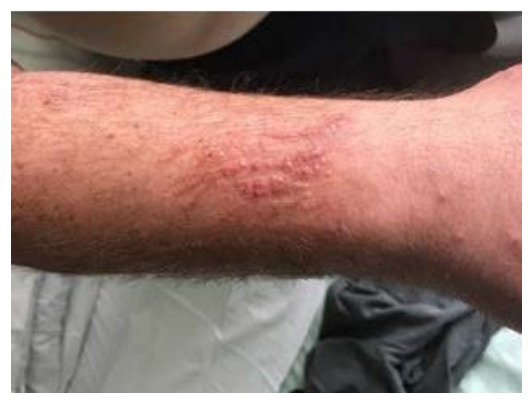

Figure 1. Erythema rashes with some scratch marks on the forearm.
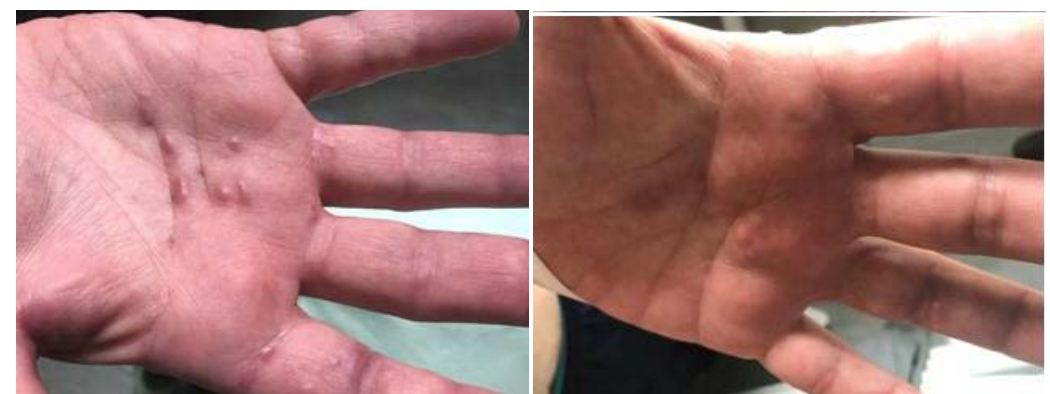

Figures 2. Some itchy vesicles on the palms. 


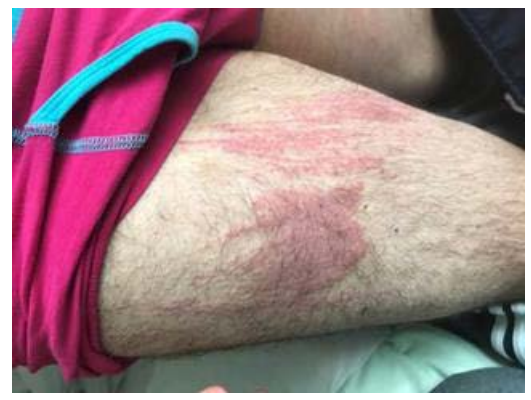

Figure 3. Erythema rashes affecting the shin area.

\section{Discussion}

Bleomycin is an antibiotic derived from the fungus Streptomyces verticillus and widely used as a chemotherapy drug. It causes DNA strand scission (breakages in the DNA strand), preventing cell replication. Bleomycin is an antitumor antibiotic chemotherapy drug used in the treatment of squamous cell cancers, some germ cell tumours, Hodgkin's and non-Hodgkin's lymphoma [1]. Bleomycin is mainly excreted from the body via the kidneys. It can also be inactivated in the body by hydrolase enzymes, the level of which varies in different tissues. There is a high rate of skin side effects when used intravenously to treat cancer because skin, in particular, has no bleomycin hydrolase activity [2]. Dermatological toxicity and mucositis are common side effects. Skin side effects develop in approximately $50 \%$ of patients receiving systemic bleomycin [3]. Serious but uncommon side effects may include vascular effects leading to heart Attack anaphylactic reactions. The main culprit is that lack of detoxifying enzymes for bleomycin in the skin makes it a vulnerable site of the adverse effects of bleomycin [4] [5] [6].

This characteristics rash may appear after administration of bleomycin by any route: intravenous, intramuscular, topical and has been reported even after intrapleural administration of the drug for management of malignant pleural effusion [7] [8] [9]. This rash may appear several hours to 2 months after the onset of administration of bleomycin [10]. Though initially believed to be associated with cumulative bleomycin dosage, several reports point out the fact that this rash is indeed dose independent and can manifest after administration of variable dose of bleomycin ranging from 5 IU to 465 IU [11].

Histological features of this drug related skin reaction are rather non-specific. Common histological changes include inconspicuous epidermal or spongiotic dermatitis, superficial lymphocytic infiltrate with neutrophil and eosinophilic granulocytes, dermal edema, melanophores in papillary dermis and epidermal hyperpigmentation [11]. Occasionally, necrotic keratinocytes and vacuolar degeneration at dermoepidermal junction may be discerned [12]. Histological changes count on the stage of evolution of skin lesions and the site of biopsy (central versus peripheral).

The plausible mechanisms for this adverse effect include localized increase in 
melanogenesis, pigmentary incontinence secondary to inflammation, alterations in normal pigmentation patterns, and toxic effects of the drug itself, inducing neutrophilic eccrine hidradenitis [13]. Histological and ultra-structural studies indicate that bleomycin reduces the epidermal turnover, resulting in a prolonged contact between melanocytes and keratinocytes [13]. It is likely that bleomycin hydrolase is expressed in human epidermis but is not able to degrade the drug efficiently. Toxic cutaneous concentration of bleomycin might be the most probable explanation for this skin eruption. It is further speculated that linear pigmentation may be caused by scratching, which induces subclinical local vasodilatation by a demographic mechanism resulting in excessive local accumulation of bleomycin.

Occurrence of bleomycin induced allergic reaction mandates prompt institution of treatment with antihistamines and topical and oral corticosteroid [14] [15]. Severe rash requires discontinuation of bleomycin. Exclusion of bleomycin in the retrospective analysis of large cohort of patients of Hodgkin's lymphoma initially treated with bleomycin containing regimen did not interfere with overall therapeutic success [16] [17] [18].

In contrast to the bleomycin induced drug reaction, patients with herpes infection may present mainly with blisters, ulcers and pain. Those symptoms usually develop about 4 days after exposure. There is no drug that can eradicate the herpes virus. However, antiviral medication such as acyclovir prevents the virus from multiplying and works very well symptomatically.

\section{Conclusion}

In summary, bleomycin induced drug allergy is one of the unwanted side effects and that can be mistaken for herpes skin infections leading clinicians' management decision to the wrong side. Careful history taken with thorough and systematic examination is of paramount importance to arrive at the correct diagnosis with appropriate management. This case report illustrates the differences in the clinical manifestation of bleomycin induced drug reaction and herpes infection and highlights the importance of thorough and systematic clinical examination that dictates the correct diagnosis with effective clinical management.

\section{Conflicts of Interest}

The authors declare no conflicts of interest regarding the publication of this paper.

\section{References}

[1] Abess, A., Keel, D.M. and Graham, B.S. (2003) Flagellate Hyperpigmentation Following Intralesional Bleomycin Treatment of Verruca Plantaris. Archives of Dermatology, 139, 337-339. https://doi.org/10.1001/archderm.139.3.337

[2] Saitta, P., Kishnamurthy, K. and Brown, L.H. (2008) Bleomycin in Dermatology: A Review of Intralesional Applications. Dermatologic Surgery, 34, 1299-1313. https://doi.org/10.1097/00042728-200810000-00001 
[3] Vanhooteghem, O., Richert, B. and de la Brassinne, M. (2001) Raynaud Phenomenon after Treatment of Verruca Vulgaris of the Sole with Intralesional Injection of Bleomycin. Pediatric Dermatology, 18, 249-251. https://doi.org/10.1046/j.1525-1470.2001.018003249.x

[4] Umezawa, H. (1965) Bleomycin and Other Antitumor Antibiotics of High Molecular Weight. Antimicrob Agents Chemother (Bethesda), 5, 1079-1085.

[5] Lazo, J.S. and Humphreys, C.J. (1983) Lack of Metabolism as the Biochemical Basis of Bleomycin-Induced Pulmonary Toxicity. Proceedings of the National Academy of Sciences of the United States of America, 80, 3064-3068. https://doi.org/10.1073/pnas.80.10.3064

[6] Chen, Y.B., Rahemtullah, A., Breeden, E. and Hochberg, E.P. (2007) Bleomycin-Induced Flagellate Erythema. Journal of Clinical Oncology, 25, 898-900. https://doi.org/10.1200/JCO.2006.09.7691

[7] Rubeiz, N.G., Salem, Z., Dibbs, R. and Kibbi, A.G. (1999) Bleomycin-Induced Urticarial Flagellate Drug Hypersensitivity Reaction. International Journal of Dermatology, 38, 140-141. https://doi.org/10.1046/j.1365-4362.1999.00344.x

[8] Moulin, G., Fière, B. and Beyvin, A. (1970) Cutaneous Pigmentation Caused by Bleomycin. Bulletin de la Société Française de Dermatologie et de Syphiligraphie, 77, 293-296.

[9] Fyfe, A.J. and McKay, P. (2010) Toxicities Associated with Bleomycin. The Journal of the Royal College of Physicians of Edinburgh, 40, 213-215. https://doi.org/10.4997/JRCPE.2010.306

[10] Vuerstaek, J.D., Frank, J. and Poblete-Gutiérrez, P. (2007) Bleomycin-Induced Flagellate Dermatitis. International Journal of Dermatology, 46, 3-5. https://doi.org/10.1111/j.1365-4632.2007.03499.x

[11] Ziemer, M., Goetze, S., Juhasz, K. and Elsner, P. (2011) Flagellate Dermatitis as a Bleomycin-Specific Adverse Effect of Cytostatic Therapy: A Clinical Histopathologic Correlation. American Journal of Clinical Dermatology, 12, 68-76. https://doi.org/10.2165/11537080-000000000-00000

[12] Mowad, C.M., Nguyen, T.V., Elenitsas, R. and Leyden, J.J. (1994) Bleomycin-Induced Flagellate Dermatitis: A Clinical and Histopathological Review. British Journal of Dermatology, 131, 700-702. https://doi.org/10.1111/j.1365-2133.1994.tb04986.x

[13] Al-Khenaizan, S. and Al-Berouti, B. (2011) Flagellate Pigmentation: A Unique Adverse Effect of Bleomycin Therapy. European Journal of Dermatology, 21, 146. https://doi.org/10.1684/ejd.2010.1213

[14] Takeda, A., Nonaka, M., Ishikawa, A. and Higuchi, D. (1999) Immunohistochemical Localization of the Neutral Cysteine Protease Bleomycin Hydrolase in Human skin. Archives of Dermatological Research, 291, 238-240. https://doi.org/10.1007/s004030050400

[15] Araki, Y., Tamura, K. and Seita, M. (1986) Side Effects of Peplomycin. Gan to Kagaku Ryoho, 13, 2446-2450.

[16] Tallon, B. and Lamb, S. (2008) Flagellate Erythema Induced by Docetaxel. Clinical and Experimental Dermatology, 33, 276-277. https://doi.org/10.1111/j.1365-2230.2007.02633.x

[17] Canellos, G.P., Duggan, D., Johnson, J. and Niedzwiecki, D. (2004) How Important Is Bleomycin in the Adriamycin + Bleomycin + Vinblastine + Dacarbazine Regimen? Journal of Clinical Oncology, 22, 1532-1533. https://doi.org/10.1200/JCO.2004.99.010 
[18] Biswas, A., et al. (2013) Bleomycin Induced Flagellate Erythema: Revisiting a Unique Complication. Journal of Cancer Research and Therapeutics, 9, 500-503.

https://doi.org/10.4103/0973-1482.119358 